\title{
Impact of obstructive sleep apnea on clinical outcomes in patients hospitalized with COVID-19
}

\author{
S. F. J. Voncken ${ }^{1}$ - T. M. H. Feron ${ }^{1}$ · S. A. J. S. Laven ${ }^{1}$ - U. Karaca ${ }^{1} \cdot$ K. Beerhorst ${ }^{2} \cdot$ P. Klarenbeek ${ }^{2}$. \\ J. M. J. A. A. Straetmans ${ }^{3}$. G. J. de Vries ${ }^{1}$ A. A. B. Kolfoort-Otte ${ }^{1}$ - M. D. de Kruif ${ }^{1}$ (D)
}

Received: 20 January 2021 / Revised: 7 August 2021 / Accepted: 11 August 2021 / Published online: 24 September 2021

(c) The Author(s), under exclusive licence to Springer Nature Switzerland AG 2021

\begin{abstract}
Purpose Data from large patient registry studies suggested an increased incidence and increased mortality in coronavirus disease-2019 (COVID-19) in patients with a history of obstructive sleep apnea (OSA). This study aimed to compare the prevalence of OSA in patients with and without COVID-19 among patients admitted to the same hospital in the same time period. In addition, the impact of OSA on clinical outcomes of COVID-19 infection was investigated.

Methods Observational cohort study. Clinical data were collected retrospectively from the complete medical records for each patient individually from March 1st 2020 to May 16th 2020.

Results A total of 723 patients were diagnosed with COVID-19 and 1161 with non-COVID-19 disease. The prevalence of OSA did not differ between these groups ( $n=49 ; 6.8 \%$ versus $n=66 ; 5.7 \% ; p=0.230)$. In patients with COVID-19, mortality was increased in the group of 49 patients with OSA $(n=17 ; 34.7 \%)$ compared to 674 COVID-19 patients without OSA $(n=143 ; 21.2 \% ; p=0.028)$. This increased risk of mortality in COVID-19 patients with OSA (OR $=2.590 ; 95 \% \mathrm{CI}$ 1.218-5.507) was independent from Body Mass Index (BMI), male gender, age, diabetes, cardiovascular disease, and obstructive lung disease. Presence of OSA in COVID-19 disease was further associated with an increased length of hospital stay $(12.6 \pm 15.7$ days versus $9.6 \pm 9.9$ days; $p=0.049)$.

Conclusion The prevalence of OSA did not differ between patients with or without COVID-19, but mortality and hospital length of stay were increased in patients with OSA and comorbid COVID-19. Hence, OSA should be included in COVID-19 risk factor analyses, Clinicians should be aware of the association and the mechanism should be further explored.
\end{abstract}

Keywords Obstructive sleep apnea $\cdot$ COVID-19 $\cdot$ Risk factor $\cdot$ Mortality

S. FJ Voncken, T. MH Feron, and S. AJS Laven contributed equally.

M. D. de Kruif

m.dekruif@ zuyderland.nl

1 Department of Pulmonology, Zuyderland Medical Center, Henri Dunantstraat 5, 6419 PC Heerlen, the Netherlands

2 Department of Neurology, Zuyderland Medical Center, Heerlen, the Netherlands

3 Department of Otorhinolaryngology and Head and Neck Surgery, Zuyderland Medical Center, Heerlen, the Netherlands

\section{Introduction}

Patients with severe Coronavirus Disease 2019 (COVID-19) share common characteristics with patients with obstructive sleep apnea (OSA). Both conditions are associated with obesity, male gender, and older age [1,2]. Also, comorbidities including diabetes mellitus, cardiovascular disease, and hypertension are linked to a worse prognosis in COVID-19 disease as well as in OSA [1, 3, 4].

Recent reports described that patients with COVID-19 and OSA showed a worse prognosis compared to COVID19 patients without OSA. These reports demonstrated an increased risk of hospitalization and death for COVID-19 patients with OSA [5-7]. In addition, a higher prevalence of OSA was observed among patients hospitalized with COVID-19 [6]. This finding was supported by a high prevalence of sleep disordered breathing measured by the Sleep 
Apnea Test (SAT) in patients during the recovery phase of COVID-19 [8]. Altogether, these findings raised concerns for many patients with OSA [9]. However, it is important to note that most data in these studies were derived from large, multicenter databases [5-7]. Despite the advantages of large-scale databases to demonstrate associations, they are vulnerable to bias $[10,11]$. In order to confirm a meaningful relationship, more detailed studies in different settings are needed. In addition, for good comparison of the prevalence of a condition between patients and a control group, data collection of both groups should be as equal as possible.

Therefore, the first aim of this study was to compare the prevalence of OSA in COVID-19 patients to non-COVID-19 patients presenting to the same Emergency Department (ED). The second aim was to investigate the contribution of OSA to mortality and other disease outcomes in a Dutch, single-center cohort with patients with a COVID-19 infection.

\section{Methods}

\section{Setting}

This study was performed at Zuyderland Medical Center, a large teaching hospital with two main sites located in Heerlen and Sittard/Geleen, the Netherlands. The study was designed as a retrospective, observational cohort study. The study was approved by the local medical ethics committee (METC-Z-20200117) and was performed in accordance with the ethical standards laid down in the 1964 Declaration of Helsinki and its later amendments. All patients received written information about the registry as well as an opt-out form to refuse use of their data at any time without any comment.

\section{Study design}

The study time period covered the peak of the 'first wave' of the COVID-19 pandemic in the Netherlands between March 1st 2020 and May 16th 2020. The data for this study were extracted from the ZuydErLand COVID-19 regiStry (ELVIS) [12]. The ELVIS was established during the COVID-19 pandemic in 2020 and includes all patients with a suspicion of COVID-19 presenting to the emergency department and admitted to Zuyderland Medical Center retrospectively. In the study period, all patients were routinely tested by naso-oropharyngeal swab for presence of severe acute respiratory syndrome coronavirus 2 (SARS-CoV-2) when they presented at the emergency department with any possible sign of COVID-19 (any respiratory complaint: dyspnea, cough, sputum or sore throat; or fever, abdominal complaints or recent contact with a confirmed COVID-19 case). After May 16th, protocols changed and patients at the emergency department were tested on indication only. For patients with multiple admissions, only the first admissions with a suspicion of COVID-19 were included. Exclusion criteria were as follows: age $<18$ years and refusal of data use by the subject. OSA was defined by an Apnea-Hypopnea Index $(\mathrm{AHI})>5.0$. In the Netherlands, diagnosis of OSA is restricted to authorized institutions only and poly(somno) graphy is the only approved method for diagnosis. Patient records were searched for a diagnosis of OSA by screening patient history, patient letters and administrative coding according to the International Classification of Diseases (ICD)-10 for each patient individually. For the patients identified with OSA, additional information, including the initial Apnea-Hypopnea Index (AHI) at time of diagnosis, Oxygen Desaturation Index (ODI) and OSA therapy, was collected from the patient records as far as possible. The patients, for whom information about the initial AHI could be retrieved, were designated patients with 'documented OSA'. Comorbidities were scored using the same approach. Cardiovascular disease was defined as a previous diagnosis of hypertension, coronary artery disease, heart rhythm abnormalities, heart failure, cerebrovascular disease, and/ or peripheral vascular disease. Obstructive lung disease was defined as a previous diagnosis of asthma and/or chronic obstructive pulmonary disease (COPD). The primary disease outcomes were mortality during admission, admission to the Intensive Care Unit (ICU), length of hospital stay (days calculated from date of admission to date of discharge) and the proportion of patients with prolonged hospital stay (defined as $>1$ week of hospital stay).

\section{Analysis}

The sample size of the study could not be based upon calculations due to the novelty of the disease and was determined by including all patients within the study time frame. The total study population was divided into two groups based on the presence of a COVID-19 diagnosis at discharge. For determination of prevalence, the group of hospitalized patients without a final diagnosis of COVID-19 at discharge was defined as the control group. The patient group diagnosed with COVID-19 was divided into two groups, based on a history with OSA. For subanalysis, the group with the combination of COVID-19 and OSA was further divided into subgroups according to 'documented OSA' only and dichotomization by AHI $>15$. Comparisons between the groups were made using SPSS software (version 24) and GraphPad Prism version 6.01. Group comparisons were performed by Student's $t$-test and Mann-Whitney $U$-test for continuous variables and Pearson's chi square and Fisher's exact for categorical variables. Kruskal-Wallis one-way ANOVA was used for comparing mortality and presence 
of OSA per BMI class. Univariate and multivariate binary logistic regression models were used to determine the predictive value of OSA, which was defined as an independent categorical variable in the analysis, adjusted by age, sex, Body Mass Index (BMI; entered as a continuous variable unless otherwise specified), and comorbidities including diabetes mellitus, cardiovascular disease, and obstructive lung disease, for prediction of adverse clinical outcomes of COVID-19 including mortality, ICU admission, and hospital stay $>1$ week. The odds ratio (OR) along with the $95 \%$ confidence interval $(95 \% \mathrm{CI})$ was reported. A $P$-value $<0.05$ was regarded as statistically significant. The variance inflation factor was calculated for all variables in the multivariate analysis to check for multicollinearity.

\section{Results}

\section{Patients}

Between March 1st 2020 and May 16th 2020, 2086 patients were screened who visited the ED with a suspicion of possible COVID-19. After applying exclusion criteria, 1884 patients were included in the study (Fig. 1). Overall, 723 patients were diagnosed with COVID-19 and 1161 were diagnosed with a diagnosis other than COVID-19. NonCOVID-19 viral pneumonia was detected in 22 of these patients.

\section{Patient characteristics and prevalence of OSA}

The patient characteristics of patients with or without COVID-19 are shown in Table 1. The overall prevalence of OSA in the group with COVID-19 did not differ significantly from the prevalence in the patient group with a diagnosis other than COVID-19 $(n=49,6.8 \%$ versus $n=66,5.7 \%$;

Table.1 Prevalence of OSA and other characteristics of hospitalized patients with or without COVID-19

\begin{tabular}{llll}
\hline Clinical features & $\begin{array}{l}\text { COVID-19 } \\
n=723\end{array}$ & $\begin{array}{l}\text { no COVID-19 } \\
n=1161\end{array}$ & $\mathrm{p}$ \\
\hline Sex (male) & $444(61 \%)$ & $634(55 \%)$ & 0.004 \\
Age (years) & $69.7 \pm 13.5$ & $68.0 \pm 15.8$ & 0.018 \\
BMI (kg/m2) & $28.1 \pm 5.6$ & $26.6 \pm 5.6$ & 0.000 \\
OSA & $49(6.8 \%)$ & $66(5.7 \%)$ & 0.355 \\
OSA (documented only) & $46(6.4 \%)$ & $51(4.4 \%)$ & 0.060 \\
Time since OSA diagnosis & $5.51 \pm 4.85$ & $5.03 \pm 3.69$ & 0.552 \\
$\quad$ years) & $167(23 \%)$ & $258(22 \%)$ & 0.658 \\
Diabetes mellitus & $441(61 \%)$ & $718(62 \%)$ & 0.713 \\
Cardiovascular disease & $168(23 \%)$ & $285(25 \%)$ & 0.517 \\
Obstructive lung disease & $37.9 \pm 1.2$ & $37.2 \pm 1.1$ & 0.000 \\
Temperature (celsius) & $93.4 \pm 19.3$ & $90.9 \pm 22.7$ & 0.014 \\
Heart rate (min-1) & $22.9 \pm 6.6$ & $19.7 \pm 5.6$ & 0.000 \\
Respiratory rate (min-1) & $107.4 \pm 16.0$ & $110.3 \pm 19.3$ & 0.001 \\
Mean arterial pressure (mmHg) & $96.5 \pm 82.3$ & $54.3 \pm 83.6$ & 0.000 \\
CRP (mg/L) & & & \\
\hline
\end{tabular}

Mean \pm SD for continuous variables and $\mathrm{n}(\%)$ for categorical variables. $S D$ standard Deviation. BMI Body Mass Index. OSA obstructive sleep apnea. $C R P$ C-reactive protein
Fig. 1 Flowchart patients were admitted to the hospital with a suspicion of Coronavirus disease 2019 (COVID-19). $\mathrm{ED}=$ emergency department. OSA $=$ obstructive sleep apnea

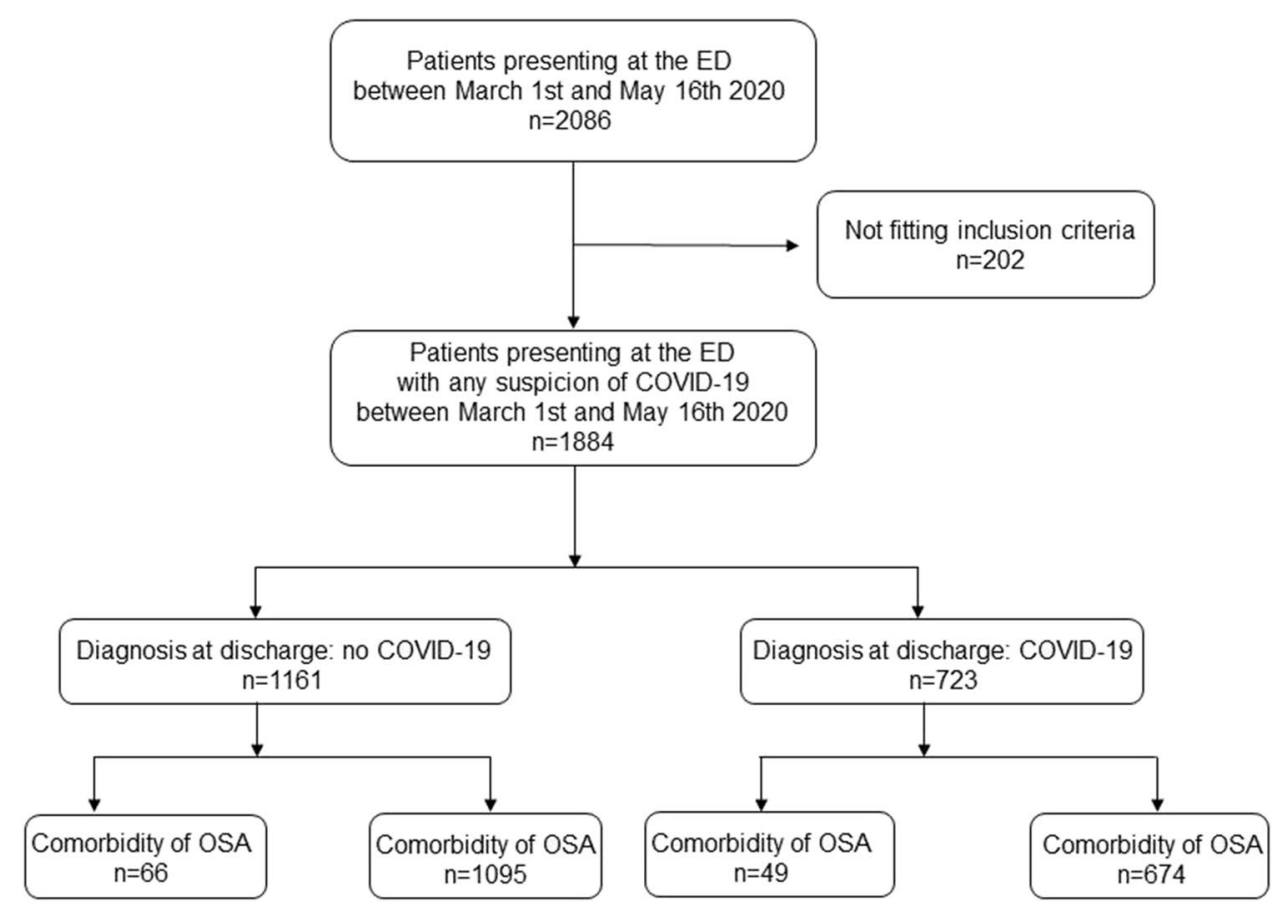


$p=0.355$ ). Original documentation about the initial AHI at the time of diagnosis of OSA was retrieved from medical records for 97 out of 115 patients. In this subgroup with 'documented OSA' only, the prevalence of OSA was not significantly different in the group with COVID-19 versus the group without COVID- 19 ( $n=46,6.4 \%$ versus $n=51$, $4.4 \% ; p=0.060)$. Further analysis in this group showed that the prevalence of OSA did not differ between groups with or without COVID-19 after stratification for AHI $>10$ ( $n=38,5.3 \%$ versus $n=45,3.9 \% ; p=0.167)$ or AHI $>15$ $(n=31,4.3 \%$ versus $n=34,2.9 \% ; p=0.121)$. Furthermore, the prevalence of OSA between patients with or without COVID-19 did not change significantly after exclusion of patients diagnosed with non-COVID-19 viral pneumonia.

Subsequently, the groups were divided according to the presence of OSA. Table 2 shows that a higher BMI and a diagnosis of diabetes mellitus were more common in patients with OSA, regardless of the presence of COVID19. In the patient group without COVID-19, a higher proportion of males was associated with OSA and in the group of patients with COVID-19, OSA patients showed a higher body temperature. In the patient subgroup with 'documented OSA' only, these same comparisons showed similar associations except for a lower age in COVID-19 patients $(65.6 \pm 10.4$ years in patients with OSA versus $70.0 \pm 13.6$ years in patients without OSA, $p=0.007$ ) and a lack of differences in diabetes mellitus and temperature in COVID-19 patients.

\section{Outcomes in COVID-19 patients and OSA}

Mortality was significantly increased in COVID-19 patients with OSA in comparison to COVID-19 patients without OSA (Fig. 2). Among the 49 OSA patients, 17 patients
(34.7\%) did not survive the COVID-19 disease compared to $143(21.2 \%)$ in the 674 COVID-19 patients without OSA $(p=0.028)$. In the subgroup with 'documented OSA' only, mortality was also increased in OSA patients $(n=16 / 46$ versus $n=144 / 677$ patients without OSA; $p=0.033$ ). Besides higher mortality rates, the COVID-19 group with OSA had a greater proportion of patients in need of intensive medical care at the ICU ( $n=24.5 \%$ versus $13.9 \% ; p=0.044)$ and an increased overall length of hospital stay $(12.6 \pm 15.7$ days versus $9.6 \pm 9.9$ days; $p=0.049$ ). Admission to ICU and length of hospital stay did not differ significantly in the subgroup with 'documented OSA' only. In a subanalysis of patients dichotomized by AHI, $10 / 31$ patients (32\%) with AHI $>15$ died, compared to $150 / 691$ patients (22\%) without $\mathrm{AHI}>15, p=0.184$.

\section{Prognostic factors in COVID-19 patients and OSA}

Univariate regression analysis of patients with COVID-19 showed that mortality was significantly predicted by higher age (OR 1.064, 95\% CI 1.046-1.083; $p<0.000$ ), OSA (OR $1.973 ; 95 \%$ CI $1.065-6.654 ; p=0.031)$ and cardiovascular disease (OR 1.723; 85\% CI 1.179-2.517), but not by male sex $(p=0.748)$, BMI $(p=0.386)$, obstructive lung disease $(p=0.803)$ and diabetes (OR 1.414, 95\% CI 0.949-2.107; $p=0.088$ ). Multivariate regression analysis results are shown in Fig. 3. Together with higher age, OSA was a significant predictor of mortality (OR 2.590; 95\% CI 1.218-5.507; in the subgroup of 'documented OSA' only OR $2.740 ; 95 \%$ CI $1.261-5.954 ; p=0.011$ ). Admission to ICU was associated with male sex and younger age. A hospital stay $>1$ week was predicted by male sex, higher age, and OSA, but not by 'documented OSA' only (OR 1.845; 95\% CI 0.947-3.596). Further analysis in the outcome prediction models showed
Table.2 Patient characteristics of OSA versus no OSA in hospitalized patients with or without COVID-19

\begin{tabular}{lllllll}
\hline & COVID-19 & & \multicolumn{3}{c}{ no COVID-19 } \\
Clinical features & with OSA & without OSA & p & with OSA & without OSA & p \\
& $\mathrm{n}=49$ & $\mathrm{n}=674$ & & $\mathrm{n}=66$ & $\mathrm{n}=1095$ & \\
\hline Sex (male) & $35(71 \%)$ & $409(61 \%)$ & 0.136 & $50(76 \%)$ & $584(53 \%)$ & 0.000 \\
Age (years) & $66.2 \pm 10.5$ & $69.9 \pm 13.6$ & 0.065 & $66.9 \pm 11.6$ & $68.1 \pm 16.0$ & 0.546 \\
BMI (kg/m2) & $33.7 \pm 8.0$ & $27.7 \pm 5.2$ & 0.000 & $31.8 \pm 5.7$ & $26.2 \pm 5.4$ & 0.000 \\
Diabetes mellitus & $17(35 \%)$ & $150(22 \%)$ & 0.046 & $30(46 \%)$ & $228(21 \%)$ & 0.000 \\
Cardiovascular disease & $29(59 \%)$ & $412(61 \%)$ & 0.788 & $48(72 \%)$ & $670(61 \%)$ & 0.061 \\
Obstructive lung disease & $13(27 \%)$ & $155(23 \%)$ & 0.572 & $17(26 \%)$ & $268(25 \%)$ & 0.814 \\
Temperature (celsius) & $38.2 \pm 1.2$ & $37.9 \pm 1.1$ & 0.045 & $37.1 \pm 1.1$ & $37.1 \pm 1.1$ & 0.584 \\
Heart rate (min-1) & $93.1 \pm 19.0$ & $93.1 \pm 19.0$ & 0.101 & $87.3 \pm 22.6$ & $91.1 \pm 22.7$ & 0.193 \\
Respiratory rate (min-1) & $22.8 \pm 6.6$ & $22.8 \pm 6.6$ & 0.787 & $20.8 \pm 5.6$ & $19.7 \pm 5.6$ & 0.128 \\
Mean arterial pressure (mmHg) & $107.1 \pm 15.8$ & $107.1 \pm 15.8$ & 0.073 & $107.2 \pm 17.3$ & $110.5 \pm 19.4$ & 0.178 \\
CRP (mg/L) & $96.2 \pm 82.3$ & $96.2 \pm 82.3$ & 0.697 & $59.2 \pm 108.0$ & $54.1 \pm 82.1$ & 0.698 \\
\hline
\end{tabular}

Mean \pm SD for continuous variables and $\mathrm{n}(\%)$ for categorical variables. SD standard deviation. BMI Body Mass Index. OSA obstructive sleep apnea. CRP C-reactive protein 
Fig. 2 Coronavirus disease 2019 (COVID-19) disease outcomes. Overview of disease severity, based on mortality, admission to ICU, total hospital stay, and hospital stay $>1$ week in COVID-19 patients with OSA compared to COVID-19 patients without OSA. ICU $=$ intensive care unit. OSA = obstructive sleep apnea. $*$ = statistically significant difference. $1=$ hospital stay more than one week ( $>7$ days). $\mathrm{NS}=$ not statistically significant
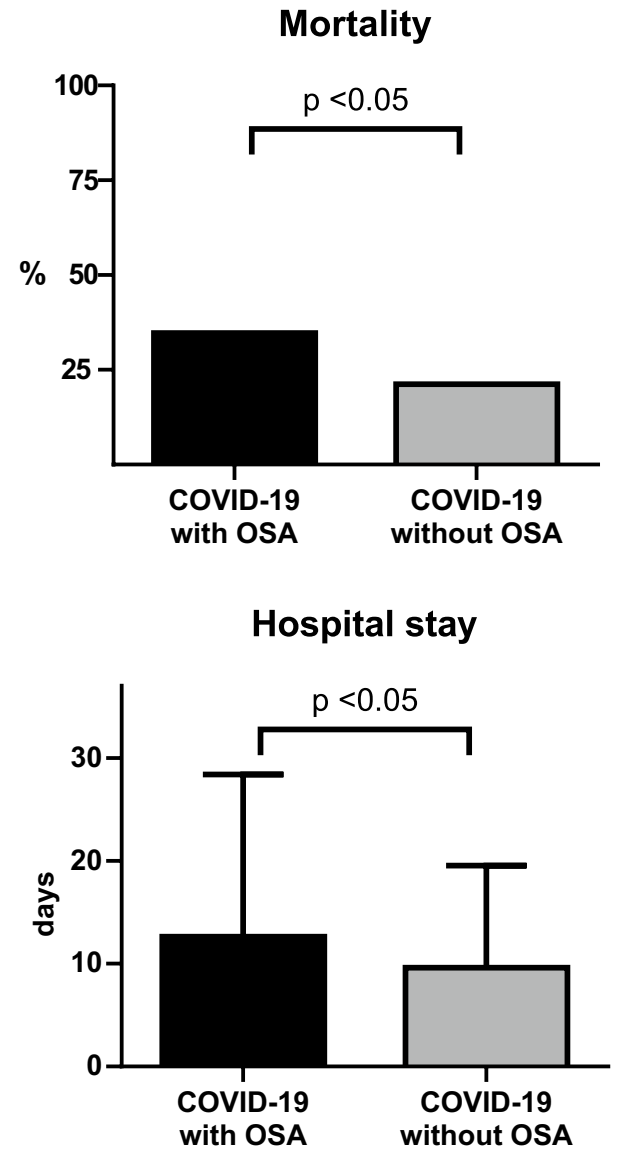

ICU admission

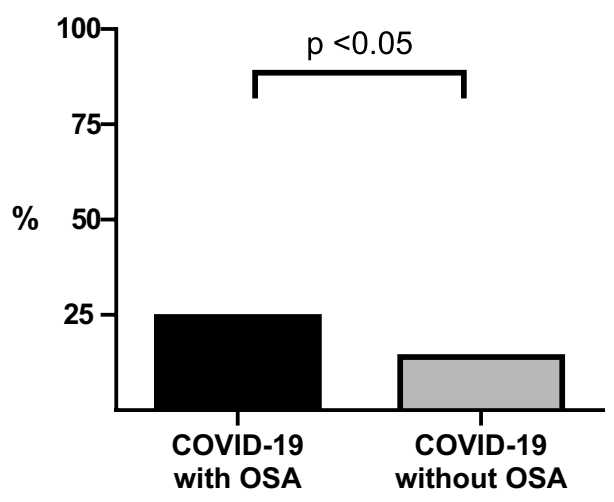

Hospital stay $>1$ week

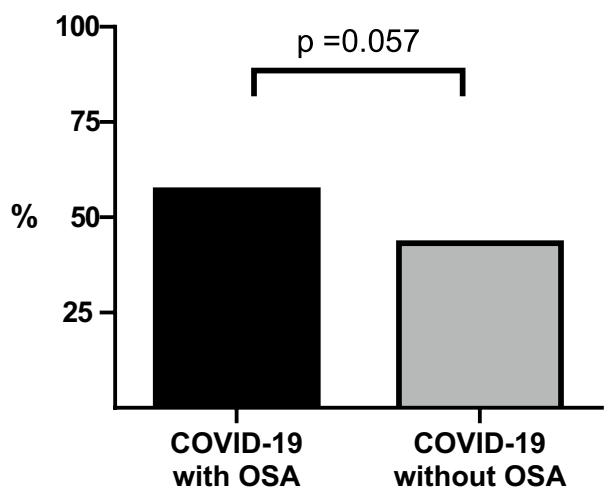

that OSA with a cut-off of AHI $>15$ predicted mortality significantly $(p=0.046)$ independently from BMI $(p=0.804)$, but did not predict ICU admission $(p=0.533)$ or hospital stay $>1$ week $(p=0.125)$.

\section{BMI and OSA and mortality in COVID-19}

Because BMI was not a significant predictor of mortality, in contrast to OSA, the possibility of extreme multicollinearity was explored. The variance inflation factor was $<2$ for all variable comparisons in the analysis, which suggests that no significant multicollinearity was present. Stratification of BMI into World Health Organization BMI classes showed a relationship between BMI class and a history of OSA $(p<0.001)$, but no association between BMI classes and mortality (Fig. 4). Dichotomization of BMI classes showed that mortality was increased in COVID-19 patients with a BMI below $25 \mathrm{~kg} / \mathrm{m} 2(n=71$ non survivors in 261 patients, $27 \%)$ compared to a BMI over $25 \mathrm{~kg} / \mathrm{m} 2(n=89$ non survivors in 462 patients; $19 \% ; p=0.014)$. When BMI was dichotomized below or over $30 \mathrm{~kg} / \mathrm{m} 2$ or $35 \mathrm{~kg} / \mathrm{m} 2$, no associations with mortality were found $(p=0.402$ and $p=0.754)$.

\section{Discussion}

The prevalence of OSA did not differ between patients with COVID-19 and other patients without COVID-19 presenting to the same emergency department in the same time period. However, a strong impact of OSA on the course of COVID19 disease was found. Mortality in COVID-19 patients with OSA was increased independently from other variables in logistic regression analysis $(\mathrm{OR}=2.590)$. Also, the length of hospital stay was increased by three days in the COVID-19 group with OSA compared to the COVID-19 group without OSA. A hospital stay $>1$ week was significantly and independently associated with OSA (OR 2.033). ICU admission was increased in COVID-19 patients with OSA, but this result was not independent from other variables in the regression analysis. Taken together, these results suggest a relevant and important contribution of OSA to mortality and hospital stay in patients with COVID-19 disease.

The prevalence of OSA in COVID-19 has been the subject of debate [10]. A previous study by Maas et al. found a prevalence of $6.3 \%$ in patients with COVID-19 in their cohort from the USA [6]. This is in concordance with the results from the present study as well as with estimations of the worldwide prevalence of OSA between 3 and $7 \%$. 
Fig. 3 Risk factor analysis of coronavirus disease 2019 (COVID-19) for clinical outcomes. $\mathrm{OR}=$ odds ratio $\mathrm{CI}=$ confidence interval. $\mathrm{BMI}=$ Body Mass Index OSA $=$ obstructive sleep apnea
Mortality

Sex (male)
Age
BMI
OSA

Diabetes Mellitus

Cardiovascular disease

Obstructive lung disease

Admission to ICU

Sex (male)

Age

BMI

OSA

Diabetes Mellitus

Cardiovascular disease

Obstructive lung disease

Hospital stay $>1$ week

Sex (male)

Age

BMI

OSA

Diabetes Mellitus

Cardiovascular disease

Obstructive lung disease
OR $(95 \% \mathrm{Cl})$

$1.236(0.813-1.879)$

$1.050(1.074-1.098)$

$1.002(0.963-1.043)$

$2.590(1.218-5.507)$

$1.316(0.827-2.094)$

$0.820(0.508-1.321)$

$0.872(0.546-1.393)$

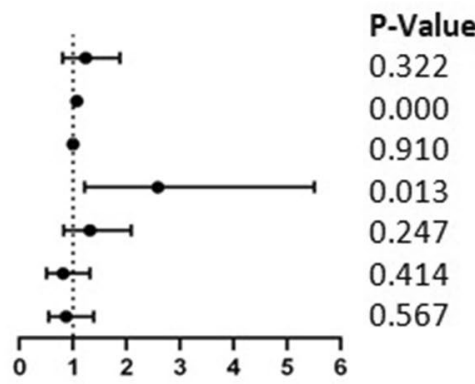

OR ( $95 \% \mathrm{Cl})$

$2.653(1.539-4.574)$

$0.980(0.961-0.999)$

$1.042(0.998-1.087)$

$1.191(0.524-2.706)$

$1.016(0.570-1.811)$

$0.751(0.435-1.296)$

$0.698(0.389-1.254)$

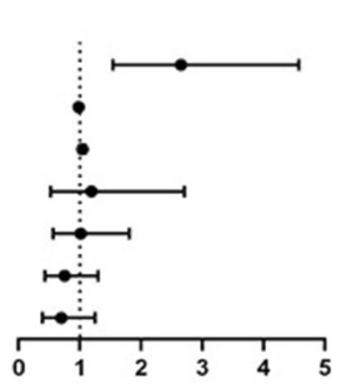

P-Value

0.000

0.041

0.060

0.677

0.957

0.304

0.229

\section{OR $(95 \% \mathrm{Cl})$}

1.402 (1.016-1.935)

1.020 (1.006-1.034)

$1.002(0.972-1.033)$

2.033 (1.055-3.918)

1.000 (0.681-1.467)

$0.698(0.481-1.011)$

$1.171(0.815-1.681)$

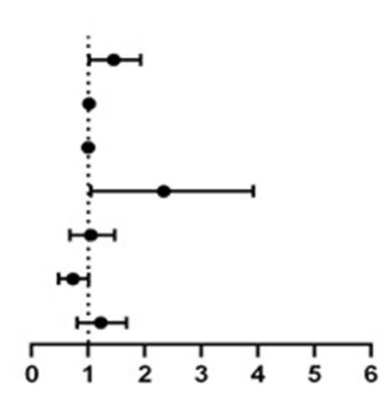

P-Value

0.040

0.006

0.911

0.034

0.999

0.057

0.392
However, because the prevalence in their control group was remarkably low with $0.8 \%$, Maas et al. concluded that the prevalence of OSA in COVID-19 was highly increased. Hence, the control group proves to be essential for proper estimation of the prevalence of OSA in COVID-19. Whereas the control group from Maas et al. was derived from historical data from an electronic hospital registry, the control group in the present study was based upon contemporary patients presenting to the same emergency department, whose patient records were searched by hand for each patient individually for presence of OSA. Apart from the study by Maas et al., also another group from Italy suggested that OSA may be highly prevalent among patients with COVID19. They showed that sleep disordered breathing (SDB) was common among 44 patients tested by SAT during the recovery phase after a hospitalization for COVID-19 [8]. However, a diagnosis of SDB by SAT has not been validated in the setting of a recovery phase from a severe pulmonary infection, which may have affected sleep quality.

The strong association of OSA with increased mortality in COVID-19 is a relatively new finding. Only recently this association with mortality was demonstrated in a brief

report studying 4,668 COVID-19 patients from an electronic patient registry from New England, USA, showing an increased all-cause mortality rate (11.7\%) compared with controls (6.9\%) [7]. This study was limited by a complex registry system of OSA, which probably gave rise to some misclassifications and attenuation of effect sizes. Another study also showed that OSA was independently associated with the risk of death on day 7 in patients hospitalized for COVID-19, but this study was limited only to COVID-19 patients also suffering from diabetes [5]. The brief report by Maas et al. mentioned above [6] did not report about mortality but they showed that OSA was associated with increased risk for hospitalization (OR 1.65) and respiratory failure (OR 1.98). As such, the associations found between OSA and poor prognostic outcomes by others previously in a few large, regional patient registries, are now supported by the more detailed patient data from a single hospital in the current study.

One interesting observation in the current study was that a higher BMI did not predict mortality in patients with COVID-19. Moreover, when data were dichotomized at $25 \mathrm{~kg} / \mathrm{m}^{2}$, mortality was even increased in the patients with 

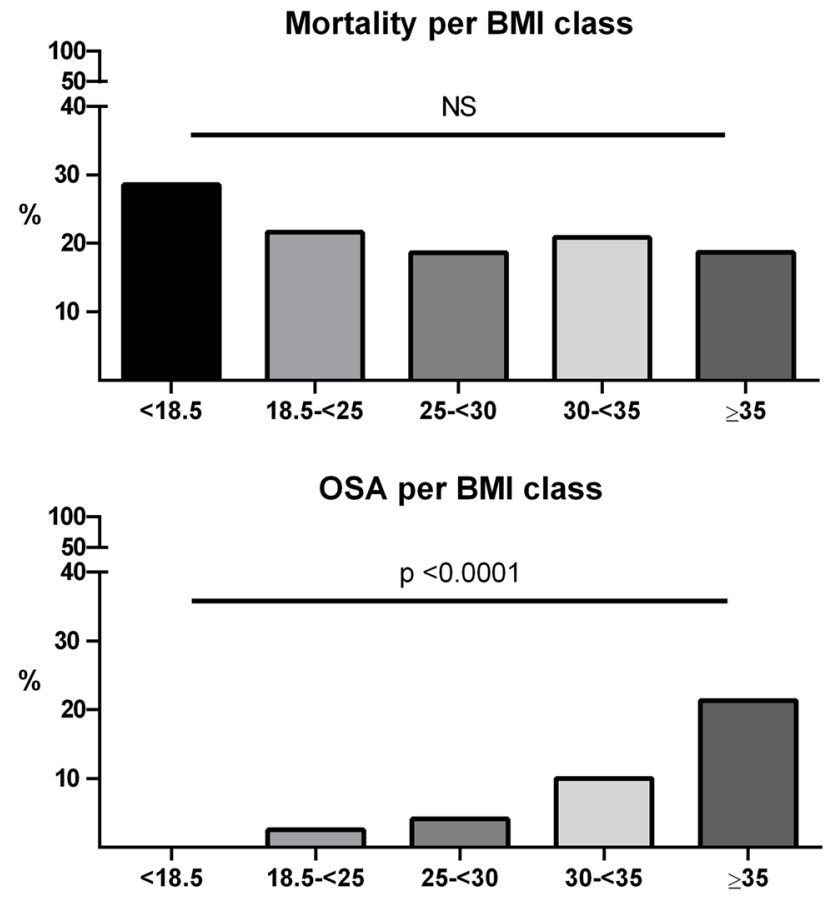

Fig. 4 Mortality and OSA per BMI class in hospitalized patients with COVID-19. BMI = Body Mass Index. OSA = obstructive sleep apnea. $\mathrm{NS}=$ non significant

a lower BMI. Although an association between a lower BMI and mortality has also been found in patients suffering from other respiratory tract viral pneumonia [13], this finding was still unexpected since other studies did show an association between a higher BMI and mortality in patients with COVID-19 [14-16]. In search of an explanation in the current study, no likely selection bias could be identified, since exclusion criteria were very few. However, some selection may have taken place by pre-hospital selection, since especially during the 'first wave' of the pandemic in the Netherlands, patients with a very low pre-hospital chance of survival were discouraged from presenting to the hospital. In contrast to the lack of association between a higher BMI and mortality in this study, OSA was a significant predictor in the regression analysis, independent from BMI. No significant multicollinearity issues were detected to help explain this difference between both variables. Because the finding is unexpected, validation by others is still required.

Several pathophysiological mechanisms may help to explain the increased mortality risk in COVID-19 patients with OSA. One frequently suggested mechanism is an increased risk of aspiration. This theory is supported by an overall higher incidence of pneumonia in OSA patients [17]. The mechanism may be related to laryngeal sensory dysfunction, laryngeal adductor reflection attenuation, impaired cough reflexes (mainly during REM sleep) and/ or gastroesophageal reflux, which have all been described in patients with OSA [18]. In addition, gasping is a common phenomenon in patients with OSA and gasping may predispose to aspiration of virus particles [19]. Furthermore, also other mechanical problems in OSA contribute to less effective ventilation treatment, including a higher risk of difficult intubation and non-invasive ventilation may be less effective due to the intermittent airway obstruction episodes [20]. In addition, higher levels of inflammatory markers have been reported in OSA patients compared to patients without OSA [21]. This could increase the risk for overproduction of early response proinflammatory cytokines which could increase chances of developing Cytokine Storm Syndrome (CSS) [22].

This study is limited by its design as a retrospective, observational study. The major pitfall in the data collection may be missing cases with undiagnosed OSA and missing cases with previously diagnosed OSA but not noted in their patient files. We tried to minimize these errors by checking the complete patient records from each patient individually and by sampling the control group from a contemporary group of patients presenting to the emergency department of the same hospital. Additional, related issues may be the reliability of OSA diagnosis when no original poly(somno) graphy data could be retrieved (for example, due to diagnosis elsewhere or loss of data during past transitions from paper to electronic patient records) and the relevance of a diagnosis of OSA with AHI $<15$ could be discussed. Therefore, additional in-depth analyses were performed in this study for subgroups with 'documented OSA' only and dichotomization by AHI. Both analyses showed no large differences with the findings in the group of OSA as a whole, which support the concept that registrations of OSA in the patient records from this study probably all represent symptomatic cases with a well-established diagnosis of OSA. Another limitation is that the diagnosis of OSA was established in the past, whereas OSA characteristics may vary in time. Therefore, the past diagnosis may not represent the actual current upper airway status of the patient at the time of hospital admission. However, the mean time since diagnosis of OSA in the current study was $5.51 \pm 4.85$ years in the COVID-19 group and $5.03 \pm 3.69$ in the patients without COVID-19, which may be considered relatively recent. Moreover, whereas OSA characteristics may vary in time, complete resolution of OSA tends to be a rare event. One final limitation is that mortality in this study referred to all-cause mortality only. This approach was chosen since it has been demonstrated that the vast majority of deaths in COVID-19 may be directly attributed to COVID-19 disease itself [23].

In conclusion, the prevalence of OSA did not differ between patients with COVID-19 and other contemporary patients at the same emergency department in this study. The data demonstrated that previous associations in large patient registry studies between OSA and adverse outcomes 
in patients with COVID-19 could be confirmed in a detailed single hospital cohort. The effect was relatively large and appeared independent from other variables including BMI. Therefore, OSA should be considered a significant risk factor to be taken into account in COVID-19 risk factor analyses, clinicians should be made aware of the association and the mechanism should be further explored.

Acknowledgements We thank Anke Linssen, Marijke Lemmens, Esther Bergman, Christel Jacquot and statistician Audrey Merry from the Bureau Wetenschappelijk Onderzoek, Mathie Leers from the department of biochemistry, and the members of the Medisch Team COVID of the Zuyderland Medical Center for their support.

Author contribution SFJV, TMHF, and SAJSL contributed equally and conceived, designed, and analyzed the study and participated in writing. UK, PK, KB, JMJAAS, and GJDV designed the study and participated in writing. AABKO designed and supervised the study and participated in writing. MDK conceived, designed, analyzed, and supervised the study and participated in writing.

Data availability The data that support the findings of this study are available on request from the corresponding author.

Code availability Not applicable.

\section{Declarations}

Ethics approval The study was approved by the local medical ethics committee (METC-Z-20200117) and was performed in accordance with the ethical standards laid down in the 1964 Declaration of Helsinki and its later amendments.

Consent to participate All patients in the ELVIS-registry received written information about the registry as well as an opt-out form in case they did not want to participate.

Consent for publication Not applicable.

Conflict of interest The authors declare no competing interests.

\section{References}

1. Zheng Z, Peng F, Xu B, Zhao J, Liu H, Peng J et al (2020) Risk factors of critical \& mortal COVID-19 cases: A systematic literature review and meta-analysis. J Infect 81(2):e16-e25. https://doi. org/10.1016/j.jinf.2020.04.021

2. Chidambaram V, Tun NL, Haque WZ, Majella MG, Sivakumar RK, Kumar A et al (2020) Factors associated with disease severity and mortality among patients with COVID-19: A systematic review and meta-analysis. PLoS ONE 15(11):e0241541. https:// doi.org/10.1371/journal.pone.0241541

3. Surani SR (2014) Diabetes, sleep apnea, obesity and cardiovascular disease: Why not address them together? World J Diabetes 5(3):381-384. https://doi.org/10.4239/wjd.v5.i3.381

4. Pinto JA, Ribeiro DK, Cavallini AF, Duarte C, Freitas GS (2016) Comorbidities Associated with Obstructive Sleep Apnea: a Retrospective Study. Int Arch Otorhinolaryngol 20(2):145-150. https:// doi.org/10.1055/s-0036-1579546
5. Cariou B, Hadjadj S, Wargny M, Pichelin M, Al-Salameh A, Allix I et al (2020) Phenotypic characteristics and prognosis of inpatients with COVID-19 and diabetes: the CORONADO study. Diabetologia 63(8):1500-1515. https://doi.org/10.1007/ s00125-020-05180-x

6. Maas MB, Kim M, Malkani RG, Abbott SM, Zee PC (2020) Obstructive sleep apnea and risk of COVID-19 infection hospitalization and respiratory failure. Sleep Breath. https://doi.org/ 10.1007/s11325-020-02203-0

7. Cade BE, Dashti HS, Hassan SM, Redline S, Karlson EW (2020) Sleep apnea and COVID-19 mortality and hospitalization. Am J Respir Crit Care Med 202(10):1462-1464. https:// doi.org/10.1164/rccm.202006-2252LE

8. Perger E, Soranna D, Pengo M, Meriggi P, Lombardi C, Parati G (2021) Sleep-disordered breathing among hospitalized patients with COVID-19. Am J Respir Crit Care Med 203(2):239-241. https://doi.org/10.1164/rccm.202010-3886LE

9. Thorpy M, Figuera-Losada M, Ahmed I, Monderer R, Petrisko M, Martin C et al (2020) Management of sleep apnea in New York City during the COVID-19 pandemic. Sleep Med 74:8690. https://doi.org/10.1016/j.sleep.2020.07.013

10. de Kruif MD, Voncken SFJ, Laven S, Feron TMH, KolfoortOtte AAB (2021) Obstructive sleep apnea and risk of COVID19 infection, hospitalization and respiratory failure. Sleep Breath. https://doi.org/10.1007/s11325-020-02271-2

11. Brault N, Saxena M (2020) For a critical appraisal of artificial intelligence in healthcare: the problem of bias in mHealth. $\mathrm{J}$ Eval Clin Pract. https://doi.org/10.1111/jep.13528

12. Ramiro S, Mostard RLM, Magro-Checa C, van Dongen CMP, Dormans T, Buijs J et al (2020) Historically controlled comparison of glucocorticoids with or without tocilizumab versus supportive care only in patients with COVID-19-associated cytokine storm syndrome: results of the CHIC study. Ann Rheum Dis 79(9):1143-1151. https://doi.org/10.1136/annrh eumdis-2020-218479

13. Jongbloed M, Leijte WT, Linssen CFM, van den Hoogen BG, van Gorp ECM, de Kruif MD (2021) Clinical impact of human metapneumovirus infections before and during the COVID-19 pandemic. Infect Dis (Lond) 53(7):488-497. https://doi.org/10. 1080/23744235.2021.1887510

14. Holman N, Knighton P, Kar P, O'Keefe J, Curley M, Weaver A et al (2020) Risk factors for COVID-19-related mortality in people with type 1 and type 2 diabetes in England: a populationbased cohort study. Lancet Diabetes Endocrinol 8(10):823-833. https://doi.org/10.1016/S2213-8587(20)30271-0

15. Alkhatib AL, Kreniske J, Zifodya JS, Fonseca V, Tahboub M, Khatib J et al (2020) BMI is associated with Coronavirus Disease 2019 intensive care unit admission in African Americans. Obesity (Silver Spring) 28(10):1798-1801. https://doi.org/10. 1002/oby.22937

16. Al-Sabah S, Al-Haddad M, Al-Youha S, Jamal M, Almazeedi S (2020) COVID-19: Impact of obesity and diabetes on disease severity. Clin Obes 10(6):e12414. https://doi.org/10.1111/cob. 12414

17. Su VY, Liu CJ, Wang HK, Wu LA, Chang SC, Perng DW et al (2014) Sleep apnea and risk of pneumonia: a nationwide population-based study. CMAJ 186(6):415-421. https://doi.org/10. 1503/cmaj. 131547

18. Chiner E, Llombart M, Valls J, Pastor E, Sancho-Chust JN, Andreu AL et al (2016) Association between obstructive sleep apnea and community-acquired pneumonia. PLoS ONE 11(4):e0152749. https://doi.org/10.1371/journal.pone.0152749

19. Beal M, Chesson A, Garcia T, Caldito G, Stucker F, Nathan CO (2004) A pilot study of quantitative aspiration in patients with symptoms of obstructive sleep apnea: comparison to a historic 
control group. Laryngoscope 114(6):965-968. https://doi.org/ 10.1097/00005537-200406000-00002

20. Nagappa M, Wong DT, Cozowicz C, Ramachandran SK, Memtsoudis SG, Chung F (2018) Is obstructive sleep apnea associated with difficult airway? Evidence from a systematic review and meta-analysis of prospective and retrospective cohort studies. PLoS ONE 13(10):e0204904. https://doi.org/10.1371/journ al.pone.0204904

21. Nadeem R, Molnar J, Madbouly EM, Nida M, Aggarwal S, Sajid $\mathrm{H}$ et al (2013) Serum inflammatory markers in obstructive sleep apnea: a meta-analysis. J Clin Sleep Med 9(10):1003-1012. https://doi.org/10.5664/jcsm.3070

22. Jose RJ, Manuel A (2020) COVID-19 cytokine storm: the interplay between inflammation and coagulation. Lancet Respir Med 8(6):e46-e47. https://doi.org/10.1016/S2213-2600(20)30216-2
23. Elezkurtaj S, Greuel S, Ihlow J, Michaelis EG, Bischoff P, Kunze CA et al (2021) Causes of death and comorbidities in hospitalized patients with COVID-19. Sci Rep 11(1):4263. https://doi.org/10. 1038/s41598-021-82862-5

Publisher's note Springer Nature remains neutral with regard to jurisdictional claims in published maps and institutional affiliations. 